\title{
SOME ALGEBRAIC PROPERTIES OF ASYMPTOTIC POWER SERIES
}

\author{
T. E. HULL
}

1. Introduction. Let us consider all power series of the form

$$
c_{0}+c_{1} z+c_{2} z^{2}+\ldots+c_{n} z^{n}+\ldots
$$

It was shown first by Borel (1) that to each such series there corresponds a non-empty class of functions such that each function in the class has the given series as its asymptotic expansion about $z=0$, the expansion being valid in a sector of the right half $z$-plane with vertex at the origin. Various generalizations of Borel's theorem have been given by Carleman (1), van der Corput (2), and Erdélyi (3).

We shall be interested only in the case where the $c_{n}$ are real and where $z$ is a real, non-negative variable $x$. We are then led to the following special case of Borel's theorem. To any series

$$
c_{0}+c_{1} x+c_{2} x^{2}+\ldots+c_{n} x^{n}+\ldots,
$$

there corresponds at least one function $f(x)$ such that

$$
R_{n}(x) x^{n}=o\left(x^{n-1}\right), \quad x \rightarrow 0,
$$

where

$$
R_{n}(x) x^{n}=f(x)-c_{0}-c_{1} x-c_{2} x^{2} \ldots-c_{n-1} x^{n-1}, \quad n=1,2,3, \ldots,
$$

is the remainder after $n$ terms.

Because of Borel's theorem the expressions "asymptotic power series" and "formal power series" are equivalent; we shall refer to them as "asymptotic series" or simply as "series." We shall refer to the class of all sum functions $f(x)$ corresponding to a particular series as the asymptotic sum of the series.

It is obvious that the collection of all asymptotic series forms a ring under formal addition, subtraction, and multiplication and it is known (4) that this ring is isomorphic to the ring of all asymptotic sums.

It is the purpose of this paper to discuss, using primarily algebraic notions, some of the properties of these rings. To do so we pay particular attention to the fundamental role played by those special asymptotic series for which

(i) $c_{0} \geqslant 0$,

(ii) there exists a sum function $f(x)$ such that, for all $x>0,\left|R_{n}(x)\right| \leqslant\left|c_{n}\right|$ $(n=0,1,2, \ldots)$, where $R_{0}(x)=f(x)$ and otherwise $R_{n}(x)$ is defined as above.

Condition (ii) means that the remainder, with respect to $f(x)$, is numerically less than the first neglected term. Any series satisfying the properties (i) and

Received June 15, 1955. This research was supported by the United States Air Force, through the Office of Scientific Research of the Air Research and Development Command. 
(ii) will be referred to as an $S$-series. Such series often arise in physical problems and because of their remainder property are especially useful in computations.

Our plan is to show first that the collection of all $S$-series is closed under formal addition and multiplication (but not subtraction). Since the distributive law will hold too, we shall call such a collection a semiring. Then we shall show that the full ring of all asymptotic series is generated from this semiring when we adjoin all differences to the semiring.

We may mention that such an imbedding of a semiring in a ring can arise in other contexts. The simplest of these is the imbedding of the semiring of all integers greater than or equal to some fixed non-negative number in the ring of all integers.

2. The S-series form a semiring. We proceed now to prove the first of our two theorems.

THEOREM 1. The S-series form a semiring. That is, the formal sum or product of two $S$-series is an $S$-series and the distributive law holds.

We show first that the coefficients in an $S$-series must alternate in sign unless the series consists of only the constant term. Suppose that $c_{n} \geqslant 0$ $(n=0,1,2, \ldots)$. Then, since

$$
R_{n}(x)=c_{n}+R_{n+1}(x) x
$$

and

we obtain

$$
\left|R_{n}(x)\right| \leqslant\left|c_{n}\right|,
$$

$$
R_{n+1}(x) x \leqslant 0
$$

so that

$$
R_{n+1}(x) \leqslant 0 .
$$

Moreover,

$$
R_{n+1}(x)=c_{n+1}+R_{n+2}(x) x
$$

and, letting $x \rightarrow 0$, we obtain

$$
R_{n+1}(0+)=c_{n+1},
$$

so that

$$
c_{n+1} \leqslant 0 .
$$

Similarly, if $c_{n} \leqslant 0$, we obtain $c_{n+1} \geqslant 0$.

We have still to show that the coefficients must all be non-zero except in the special case where the series consists of only the constant term. We obviously cannot have any coefficient equal to zero unless the series terminates; but the series cannot terminate with the term $c_{n} x^{n}(n=1,2,3, \ldots)$, because if it did we would have

$$
R_{n-1}(x)=c_{n-1}+c_{n} x
$$


and $x$ could always be chosen so large that

$$
\left|R_{n-1}(x)\right|>\left|c_{n-1}\right| \text {. }
$$

From now on we shall denote the non-constant $S$-series by, for example,

$$
f^{\alpha}=\alpha_{0}-\alpha_{1} x+\alpha_{2} x^{2}-\ldots+(-1)^{n-1} \alpha_{n-1} x^{n-1}+(-1)^{n} R_{n}{ }^{\alpha} x^{n}
$$

and

$$
f^{\beta}=\beta_{0}-\beta_{1} x+\beta_{2} x^{2}-\ldots+(-1)^{n-1} \beta_{n-1} x^{n-1}+(-1)^{n} R_{n}{ }^{\beta} x^{n},
$$

where

$$
\alpha_{n}, \beta_{n}>0 \text { and } R_{n}{ }^{\alpha}, R_{n}{ }^{\beta} \geqslant 0 .
$$

The Theorem requires us to show that the sum or product of two $S$-series is an $S$-series. The "sum" part of the proof is trivial. The "product" part is also trivial if one or both of the series is constant; for the other case we note that the remainder, with respect to $f^{\alpha} f^{\beta}$, after $n$ terms in the formal product of the above two series can be written

$$
(-1)^{n+1}\left(\alpha_{0} R_{n}{ }^{\beta}+\alpha_{1} R_{n-1}{ }^{\beta}+\ldots+\alpha_{n-1} R_{1}{ }^{\beta}+R_{n}{ }^{a} R_{0}{ }^{\beta}\right) x^{n+1}
$$

while the $(n+1)$ th term in the formal product is

$$
(-1)^{n+1}\left(\alpha_{0} \beta_{n}+\alpha_{1} \beta_{n-1}+\ldots+\alpha_{n} \beta_{0}\right) x^{n+1} \text {. }
$$

The first of these two expressions is numerically less than or equal to the second so that condition (ii) is satisfied. The first term in the formal product is $\alpha_{0} \beta_{0}>0$ and so condition (i) is also satisfied. The product series is therefore an $S$-series with respect to the function $f^{\alpha} f^{\beta}$. It is obvious that the distributive law holds and so the Theorem is proven.

In fact we have shown that the semiring of all $S$-series is isomorphic to the semiring whose elements are the classes of sum functions which satisfy condition (ii). The semiring possesses a unit and a zero element which are simply the numbers 1 and 0 respectively.

It can also be shown that the formal substitution of an $S$-series in place of the variable in a convergent series produces another $S$-series, provided the coefficients of the convergent series are positive and its radius of convergence is greater than the constant term in the first $S$-series.

Incidentally, the non-constant $S$-series alone form a semiring without, of course, either a unit or a zero element. This semiring is an ideal, if differences are not allowed, in the larger semiring of all $S$-series.

3. The semiring generates the ring. We shall now show that the full ring of all asymptotic series is generated from the semiring of all $S$-series when we adjoin all differences to the semiring. The result can be formulated in the following way.

THEOREm 2. Any asymptotic series can be written as the difference between two S-series. 
Suppose that $\alpha_{0}, \alpha_{1}>0$ and consider the series

$$
\alpha_{\theta}-\alpha_{1} x+\alpha_{2} x^{2}-\ldots+(-1)^{n} \alpha_{n} x^{n} \ldots
$$

We shall show shortly that, if $\alpha_{n+1} / \alpha_{n} \rightarrow \infty$ as $n \rightarrow \infty$ and the inequalities

$$
\alpha_{n+1} / \alpha_{n} \geqslant \alpha_{n} / \alpha_{n-1}, \quad n=1,2,3, \ldots,
$$

are also satisfied, the series is an $S$-series. The proof of the Theorem is then straightforward; for, given any series

$$
c_{0}+c_{1} x+c_{2} x^{2}+\ldots+c_{n} x^{n}+\ldots,
$$

one can always choose some $\alpha_{0}, \alpha_{1}, \beta_{0}, \beta_{1}>0$ so that $\alpha_{0}-\beta_{0}=c_{0}$ and $\alpha_{1}-\beta_{1}=-c_{1}$. Then one can always choose pairs $\alpha_{2}, \beta_{2}>0, \alpha_{3}, \beta_{3}>0, \ldots$ in turn so that $\alpha_{n+1} / \alpha_{n}$ and $\beta_{n+1} / \beta_{n} \rightarrow \infty$ as $n \rightarrow \infty$, and

$$
\frac{\alpha_{n+1}}{\alpha_{n}} \geqslant \frac{\alpha_{n}}{\alpha_{n-1}}, \quad \frac{\beta_{n+1}}{\beta_{n}} \geqslant \frac{\beta_{n}}{\beta_{n-1}}, \quad n=1,2,3, \ldots
$$

and so that $\alpha_{n}-\beta_{n}=(-1)^{n} c_{n}$. The $\alpha$-series and the $\beta$-series so formed are then both $S$-series and their difference is the given series. The Theorem is then proven.

We have only to show that the conditions assumed for $\alpha_{n}$ in the above paragraph ensure that the corresponding $\alpha$-series is an $S$-series. For a series to be an $S$-series, conditions (i) and (ii) must be satisfied. Condition (i) is satisfied since we have assumed that $\alpha_{0}>0$; in fact our assumptions guarantee that all $\alpha_{n}>0$. We can show that condition (ii) is also satisfied by constructing the required "sum" function.

We define the intervals $I_{n}$ in the following way: $I_{0}$ is the interval $0 \leqslant x \leqslant \infty$ and $I_{n}(n=1,2,3, \ldots)$ is the interval $0 \leqslant x<\alpha_{n-1} / \alpha_{n}$. Putting

$$
\mu_{n}(x)=\left\{\begin{array}{l}
1, x \in I_{n} \\
0, \quad x \notin I_{n}
\end{array}\right.
$$

we define

$$
f(x)=\sum_{i=0}^{\infty}(-1)^{i} \mu_{i}(x) \alpha_{i} x^{i}
$$

This series converges for all $x$-in fact, it terminates for each $x$. Therefore $f(x)$ is defined.

For our purposes the essential points are the following. For each $x$, the terms which appear in the series for $f(x)$ decrease in magnitude with increasing subscript (unless only the first term appears). The terms which do not appear in the series for $f(x)$ are non-decreasing in magnitude with increasing subscript.

Then, if we suppose that $x \in I_{N}-I_{N+1}(N=0,1,2, \ldots)$ and if we take account of the fact that all terms are alternating in sign, we can easily pick 
out one term which dominates $R_{n+1}(x) x^{n+1}(n=-1,0,1, \ldots)$. We obtain

$$
\left|R_{n+1}(x) x^{n+1}\right| \begin{cases}\leqslant \alpha_{n+1} x^{n+1}, & n<N, \\ =0, & n=N, \\ \leqslant \alpha_{n} x^{n}, & n>N .\end{cases}
$$

The last expression is, in turn, $\leqslant \alpha_{n+1} x^{n+1}$ when $n>N$, so that condition (ii) is satisfied. (If $N=\infty$ we of course need to consider only the case where $n<N$, and if $N=0$ we need to consider only the cases where $n \geqslant N$.)

The conditions assumed for $\alpha_{n}$ are not necessary for the series to be an $S$-series; this can be seen by considering the expansion of $e^{-x}$. Moreover it can be shown that the $S$-series which do satisfy these conditions are closed under addition but not under multiplication.

Incidentally we have in fact shown that the semiring of non-constant $S$-series also generates the full ring with the adjoining of all differences.

4. Concluding remarks. D. C. Murdoch has pointed out that the above results enable one to define a partial ordering on the ring of all power series. One series $a(x)$ can be defined to be "greater than or equal to" another series $b(x)$ if and only if their difference is an $S$-series. By using a procedure analogous to that used in the first part of Theorem 2, one can then always construct an upper bound and a lower bound to any pair of series. However, it is also possible to show that neither the least upper bound nor the greatest lower bound required for a lattice can exist.

Algebraic and other properties of asymptotic series have been considered by Popken (5). He discusses the ring of all asymptotically finite functions (and so does not restrict his attention to power series) and he shows, for example, that this ring is complete with respect to a certain non-Archimedean pseudo-valuation.

We wish to thank B. N. Moyls for many interesting discussions during the preparation of this paper, and also the referee, particularly for pointing out an error in the proof of Theorem 2.

\section{REFERENCES}

1. T. Carleman, Les fonctions quasianalytiques (Paris, 1926).

2. J. G. van der Corput, Asymptotic expansions I. Fundamental theorems of asymptotics (Univ. of California, Berkeley, 1954).

3. A. Erdélyi, Asymptotic expansions (Cal. Tech., Pasadena, 1955).

4. H. Poincaré, Sur les intégrales irregulières des équations linéaires, Acta Math., 8 (1886) 295-344.

5. J. Popken, Asymptotic expansions from an algebraic standpoint, Nederl. Akad. Wetensch. Proc., Ser. A., 56 (Indagationes Math., 15 (1953), 131-143).

University of British Columbia 